\title{
Desempeño de diferentes índices de vegetación de Sentinel-2A para estimar el rendimiento de soja en agricultura de precisión
}

\author{
Ovando, G., de la Casa, A., Díaz, G., Díaz, P., Bressanini, L. y Miranda, C.
}

\section{RESUMEN}

La agricultura de precisión (AP) apunta a identificar la variabilidad del cultivo y del suelo en un lote para mejorar el manejo y optimizar el empleo de insumos. Los mapas de rendimiento son herramientas relevantes para planificar la AP. Los índices de vegetación (IV) provenientes de la teledetección permiten monitorear la variación espacio-temporal de los cultivos durante la estación de crecimiento. El objetivo del presente trabajo fue evaluar la idoneidad de diferentes IV de Sentinel-2A para estimar el rendimiento de soja (Glycine max (L.) Merril) en el marco de la AP. El estudio se realizó empleando un mapa de rendimiento de soja (campaña 2017-2018) de un lote ubicado al sur de la ciudad de Córdoba, Argentina. Se calcularon IV empleando la diferencia, el cociente y la diferencia normalizada, tomando de a dos bandas de una imagen de Sentinel-2A del 4/2/2018. Se computó el valor absoluto del coeficiente de correlación lineal de Pearson $(|r|)$ entre los distintos IV y el rendimiento de soja. El mayor valor de $|r|(0,726)$ correspondió a la diferencia entre las bandas 8 (NIR) y 12 (SWIR), permitiendo reproducir con suficiente precisión y anticipación la variabilidad espacial de los rendimientos en el lote.

Palabras clave: teledetección, mapa de rendimiento, coeficiente de correlación lineal de Pearson

Ovando, G., de la Casa, A., Díaz, G., Díaz, P., Bressanini, L. and Miranda, C., 2021. Performance of different Sentinel-2A vegetation indices to estimate soybean yield in precision farming. Agriscientia 38 (2): $1-12$

\section{SUMMARY}

Precision agriculture (PA) aims to identify crop and soil variability to improve management and optimize the use of inputs. Yield maps become a relevant tool for PA planning. Vegetation indices (VI) from remote sensing allow monitoring the spatio-temporal variation of crops in the growing season. The objective 
of this work was to evaluate the performance of different Sentinel-2A VIs to estimate soybean (Glycine max (L.) Merril) yield within the framework of PA. The study was carried out using a soybean yield map during the $2017 / 2018$ season from a plot located to the south of Córdoba city, Argentina. Every two bands were taken from a Sentinel-2A image from 4/Feb/2018 and VI were calculated using differences, ratios and normalized differences. The absolute value of the Pearson linear correlation coefficient $(|r|)$ between the different IVs and soybean yield was computed. The highest value of $|r|(0.726)$ corresponded to the difference between bands 8 (NIR) and 12 (SWIR), allowing to reproduce with sufficient precision the spatial variability of yields in the plot.

Key words: remote sensing, yield map, Pearson linear correlation coefficient

Ovando, G. (ORCID: 0000-0002-6015-404X), de la Casa, A. (ORCID: 0000-0003-4317-6797), Díaz, G. (ORCID: 0000-0002-3714-4652), Díaz, P. (ORCID: 0000-0003-0201-349X), Bressanini, L. Universidad Nacional de Córdoba, Facultad de Ciencias Agropecuarias. Felix Marrone 746, Ciudad Universitaria, Córdoba, Argentina. CP: 5000. Miranda, C. Establecimiento "Las Delicias". Correspondencia a: gugovan@agro.unc.edu.ar

\section{INTRODUCCIÓN}

La agricultura de precisión (AP) emplea diversas herramientas y tecnologías para identificar la variabilidad del cultivo y del suelo a escala del lote, en pos de mejorar las prácticas de manejo y optimizar el empleo de los insumos agropecuarios. La variación del rendimiento en un lote no solo refleja los cambios en el potencial productivo de un área determinada, sino que también indica aquellos sectores donde una práctica de manejo en particular ha generado los resultados esperados. De esta manera, los mapas de rendimiento no sólo proveen información sobre la capacidad productiva de un terreno agrícola, sino que representan una herramienta relevante para el manejo de los sistemas agrícolas de precisión. La teledetección, que tradicionalmente ha empleado las regiones del espectro electromagnético correspondientes a la luz visible y al infrarrojo, constituye en la actualidad un componente más de la AP para realizar el monitoreo del suelo y los cultivos (Bottega de Queiroz et al., 2017; Khanal et al., 2017).

Los datos radiométricos con alta resolución espacial y temporal provenientes de la teledetección presentan un potencial para describir la variación espaciotemporal de los parámetros biofísicos de los cultivos. En tal sentido, la reciente disponibilidad de datos de imágenes de Sentinel-2, con mejor resolución espacial que Landsat 8 y bandas más específicas para identificar distintas propiedades de la vegetación, ofrece nuevas perspectivas para el monitoreo de cultivos, en particular orientadas a la escala de lote que demandan las aplicaciones de la AP (Battude et al., 2016).

Un gran número de técnicas se han desarrollado para derivar variables biofísicas del cultivo a partir de la teledetección. En general, ellas pueden agruparse en tres amplias categorías: i) la inversión de modelos de transferencia radiativa (Nigam et al., 2017); ii) el empleo de técnicas de machine learning, por ejemplo, redes neuronales (Xie et al., 2019), y iii) el empleo de índices de vegetación (IV). Los métodos basados en IV tienen el beneficio de ser computacionalmente simples y más universalmente aplicables que los otros métodos ya que son generalmente menos sitio-específico. Además, por su simpleza facilita el análisis de tipo exploratorio (Frampton et al., 2013).

Entre la gran diversidad de IV que se han desarrollado en los últimos años, el Normalized Difference Vegetation Index (NDVI) (Rouse et al., 1973) probablemente sea el que alcanzó mayor difusión en estudios sobre la vegetación. Sin embargo, numerosas investigaciones han demostrado que el NDVI resulta insensible para detectar cambios en la biomasa de vegetación cuando su densidad es moderada y alta (Viña et al., 2004). En tal sentido, Crusiol et al. (2017) consideraron la influencia que diferentes factores (momentos y alturas de medición, tamaño de muestreo y niveles de estrés hídrico) ejercen sobre las lecturas de NDVI obtenidas de sensores 
portátiles en distintos cultivares de soja en invernadero y también en pruebas de campo.

Un número creciente de estudios se han centrado en evaluar los IV en términos de su sensibilidad a los parámetros biofísicos de la vegetación, así como a los factores externos que afectan la reflectancia del canopeo (Haboudane et al., 2004; Xue y Su, 2017). Por lo tanto, es importante seleccionar las bandas más apropiadas para generar nuevos índices espectrales de carácter específico que permitan el monitoreo de parámetros biofísicos de cultivos y determinar su precisión (Tanaka et al., 2015). En este sentido, Xu et al. (2020) encontraron que el Normalized Difference Water Index (NDWI) calculado de una fusión de datos provenientes de Landsat 8 y MODIS, presentó valores del coeficiente de determinación $\left(R^{2}\right)$ de 0,85 cuando se correlacionó con el contenido hídrico de canopeos de soja. Kovar et al. (2019) confirmaron que el Photochemical Reflectance Index (PRI), calculado a partir de información de un espectro radiómetro portátil, resultó un buen estimador del contenido de agua de hojas de soja sometidas a una deshidratación rápida.

Los índices de vegetación que emplean la reflectancia en el borde rojo (red edge-aproximadamente entre 0,7 y $0,8 \mu \mathrm{m}$ ) permiten estimar el contenido de clorofila y nitrógeno de la vegetación y de esa manera monitorear la salud y el desarrollo de la vegetación. El instrumento MSI (Multi-Spectral Instrument) a bordo del satélite Sentinel-2, permite obtener información del borde rojo con una muy buena resolución espacial de $20 \mathrm{~m}$ (Clevers y Gitelson, 2013; Frampton et al., 2013).

Li et al. (2018) evaluaron diferentes IV en maíz y encontraron que red-edge-position (REP) fue más sensible a las condiciones hídricas del cultivo, potencial agua de la hoja, índice de área foliar y contenido de clorofila. Por su parte, Peng et al. (2017) encontraron que los IV que emplean la porción del borde rojo para estimar el contenido de clorofila en maíz y soja, fueron poco afectados por la fenología del cultivo. Viña y Gitelson (2005) emplearon el denominado red-edge NDVI (que reemplaza la banda roja por la red-edge) y el Wide Dynamic Range Vegetation Index, y determinaron que estos índices fueron sensibles a la variación de la fracción de la radiación fotosintéticamente activa absorbida (fAPAR) a lo largo de todo su rango, mostrando además un incremento significativo en la sensibilidad a fAPAR.

La mayoría de los IV espectrales pueden clasificarse en cinco categorías (Lu et al., 2015):

- Reflectancia de una sola banda o índices de diferencias entre bandas simple (SD)
- Índices de cocientes simples (SR)

- Índices de diferencia normalizada (ND)

- Índices que utilizan las derivadas de las reflectancias

- Otras formas de índices

(ejemplo: $\mathrm{R}_{705} /\left(\mathrm{R}_{717}+\mathrm{R}_{491}\right)$ )

Donde los valores de los subíndices indican la longitud de onda $(\mathrm{nm})$ de las reflectancias.

El objetivo de este trabajo fue evaluar el desempeño predictivo de diferentes índices de vegetación obtenidos a partir de la información radiométrica de Sentinel-2A, y en el marco de la agricultura de precisión, establecer su capacidad para estimar la variación del rendimiento de soja (Glycine max (L.) Merril) en un lote de producción en la campaña 2017-2018.

\section{MATERIALES Y MÉTODOS}

\section{Área de estudio}

El estudio se realizó a partir de información relevada en un lote de aproximadamente 48 ha (550 m x $870 \mathrm{~m}$ ) del establecimiento "Las Delicias" ubicado al sur de la ciudad de Córdoba, Argentina

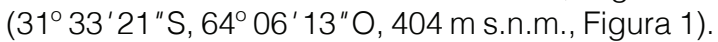
El suelo del lote corresponde a un Haplustol éntico, serie Manfredi, cuyo perfil presenta tres horizontes de textura franco-limosa (Dardanelli et al., 1997).

El 22/11/2017 este lote fue sembrado con soja de la variedad Don Mario 40R16, con una distancia entre hileras de 0,35 $\mathrm{m}$ y una densidad de $30 \mathrm{pl} \mathrm{m}^{-2}$; el cultivo fue cosechado el 30/03/2018 empleando un monitor de rendimiento John Deere GS3.

Durante el ciclo de la soja se extrajeron muestras de suelo para determinar el contenido de humedad por gravimetría (a 10, 30, 50, 70 y $90 \mathrm{~cm}$ de profundidad) en diez repeticiones tomadas al azar,

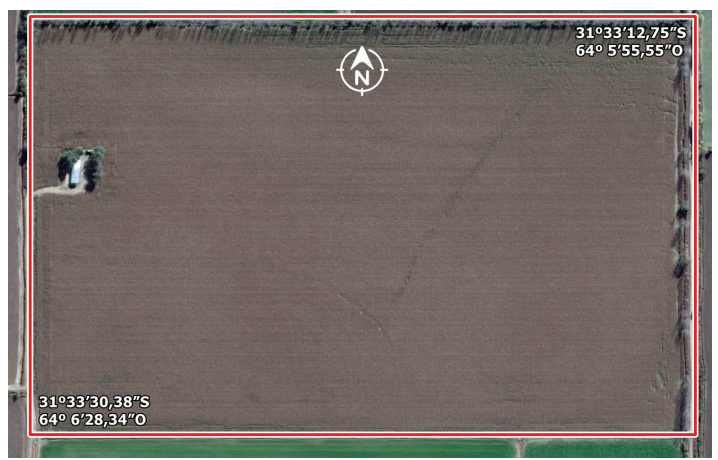

Figura 1. Imagen del lote de soja al sur de la ciudad de Córdoba, Argentina. Fuente: Google Maps 
en las fechas 26/12/2017 y 6/02/2018. El contenido de agua útil se calculó hasta 1,5 $\mathrm{m}$ de profundidad considerando los valores de capacidad de campo y de punto de marchitez permanente del suelo de Manfredi (Dardanelli et al., 1997), un único valor de densidad aparente de $1,25 \mathrm{~g} / \mathrm{cm}^{3}$ (Rollán y Bachmeier, 2014). Las fases fenológicas fueron observadas siguiendo la escala propuesta por Fehr y Caviness (1977).

\section{Mapa de rendimiento}

El mapa de rendimiento original contaba con 48.788 puntos de rendimiento en total, lo que nos lleva a una resolución de aproximadamente 9,807 $\mathrm{m}^{2}$ por punto de rendimiento. En primera instancia, las coordenadas geográficas se transformaron a coordenadas UTM (HS zona 20), y luego los datos se depuraron para descartar outliers. Se utilizó la función del semivariograma de tipo exponencial, con la que se obtuvo el mejor ajuste a través del método de mínimos cuadrados ponderados (WLS), de esta forma se estimaron los parámetros varianza nugget o efecto pepita, la varianza estructural o partial sill y el rango. Luego se realizó una interpolación espacial Kriging ordinario/universal sin tendencia, utilizando una grilla de predicción de $10 \times 10 \mathrm{~m}$, y se incorporaron los parámetros obtenidos del semivariograma; todo este procedimiento se realizó con el software GeoDa V. 1.18 (Anselin et al., 2006).

\section{Imagen de Sentinel}

En este estudio se utilizó una imagen del satélite Sentinel-2A, correspondiente al día 4/02/2018, cuando el cultivo presentaba cobertura completa. La imagen fue descargada de https:// scihub.copernicus.eu/dhus/\#/home. Bolton y Friedl (2013) y Petersen (2018) determinaron correlaciones más elevadas entre índices de vegetación y el rendimiento, cuando el cultivo estuvo completamente desarrollado. En tal sentido, Mladenova et al. (2017) encontraron un pico del valor de correlación entre el rendimiento de soja y los índices de vegetación NDVI, EVI y de profundidad óptica de la vegetación (VOD) durante el mes de agosto (para el hemisferio norte), lo que coincide con el momento de mayor requerimiento hídrico del cultivo y el período más crítico en la formación del rendimiento. Se empleó información del sensor Multi-Spectral Imager (MSI) con nivel de procesamiento L1C, que corresponde a reflectancias en el tope de la atmósfera (TOA) en geometría cartográfica; se utilizó el gránulo de $100 \times 100 \mathrm{~km}$ (tile) correspondiente a la órbita relativa 110, tile 20JLL. Sentinel $2 \mathrm{~A}$ es un satélite de órbita polar superespectral de alta resolución, con un período de revisita de 10 días. El sensor MSI a bordo tiene un ancho de barrido de $290 \mathrm{~km}$ y provee un conjunto de 13 bandas espectrales (Tabla 1), desde el espectro visible pasando por el infrarrojo cercano (NIR) hasta el infrarrojo de onda corta (SWIR) (Xie et al., 2019).

Para corregir el efecto de la atmósfera y obtener el producto L2A (reflectancia en la base de la atmósfera (BOA)) se utilizó el procesador Sen2Cor que opera dentro del software SNAP Toolbox V 6.0, empleando las bandas 1, 9 y 10 para efectuar la corrección atmosférica (Louis et al., 2016). Al efectuar la corrección atmosférica el software remuestrea los píxeles de las bandas con resoluciones espaciales diferentes a $10 \mathrm{~m}$, llevándolas a una resolución de $10 \mathrm{~m}$.

A partir de la combinación del conjunto de

Tabla 1. Características de las bandas del sensor MSI de Sentinel-2A

\begin{tabular}{ccccc}
\hline Banda & Nombre & Centro de Banda $(\mathrm{nm})$ & Ancho de Banda $(\mathrm{nm})$ & Resolución Espacial $(\mathrm{m})$ \\
\hline B1 & Coastal aerosol & 443 & 20 & 60 \\
B2 & Blue & 490 & 65 & 10 \\
B3 & Green & 560 & 35 & 10 \\
B4 & Red & 665 & 30 & 10 \\
B5 & Red edge 1 & 705 & 15 & 20 \\
B6 & Red edge 2 & 740 & 15 & 20 \\
B7 & Red edge 3 & 783 & 20 & 20 \\
B8 & NIR & 842 & 115 & 10 \\
B8A & Narrow NIR & 865 & 20 & 20 \\
B9 & Water Vapour & 945 & 20 & 60 \\
B10 & SWIR Cirrus & 1375 & 30 & 60 \\
B11 & SWIR 1 & 1610 & 90 & 20 \\
B12 & SWIR 2 & 2190 & 180 & 20 \\
\hline
\end{tabular}


las bandas tomadas de a dos, se calcularon los diferentes índices de vegetación y se computó el valor absoluto del coeficiente de correlación lineal de Pearson $(|r|)$ entre cada uno de los distintos IV y el rendimiento del cultivo de soja. Este cálculo se realizó con el software Visual Basic for Applications (VBA) de Microsof Office Excel $2007 \AA$, para un total de 3714 muestras.

Los índices de vegetación de las diferencias $(\mathrm{SD})$, cocientes (SR) y diferencias normalizadas (ND), se calcularon con las siguientes expresiones:

$$
\begin{aligned}
& \mathrm{SD}=\rho_{\lambda i}-\rho_{\lambda j} \\
& \mathrm{SR}=\frac{\rho_{\lambda i}}{\rho_{\lambda j}} \\
& \mathrm{ND}=\frac{\left(\rho_{\lambda i}-\rho_{\lambda j}\right)}{\left(\rho_{\lambda i}+\rho_{\lambda j}\right)}
\end{aligned}
$$

Donde $\lambda i$ y $\lambda \mathrm{j}$ son las reflectancias de las bandas iy $j$, respectivamente.

A partir de los índices con mejor desempeño de cada categoría, se procedió a generar un modelo lineal para estimar el rendimiento de soja y a partir de él calcular los errores, la raíz cuadrada del error cuadrático medio (RMSE) y su valor porcentual respecto del valor medio observado (\%RMSE).

\section{RESULTADOS Y DISCUSIÓN}

Como se indicó en la introducción, muchos índices de vegetación son sensibles al estado hídrico del cultivo, motivo por el cual resulta relevante analizar las condiciones meteorológicas durante el ciclo del cultivo de soja. Los datos fueron obtenidos de una estación meteorológica próxima al lote en estudio y los valores de la radiación solar astronómica (RSA, $\left.\mathrm{MJm}^{-2} \mathrm{~d}^{-1}\right)$ se calcularon siguiendo a Allen et al. (1998). Como puede observarse en la Figura 2, tanto los valores de temperatura máxima y mínima como los de radiación solar astronómica presentan sus valores más elevados durante las etapas vegetativas del cultivo y comienzan a declinar durante las fases reproductivas. Los $286 \mathrm{~mm}$ de precipitación registrada durante el ciclo del cultivo presentan una distribución irregular: se contabilizaron $224 \mathrm{~mm}$ en la primera mitad del ciclo y solamente $62 \mathrm{~mm}$ en la segunda, cuando el cultivo desarrolla la etapa reproductiva. La campaña 2017-2018 presentó una condición de sequía extrema si se considera que la precipitación promedio para el cuatrimestre desde diciembre a marzo, durante el período 19702018, para la estación Córdoba Aero dependiente del Servicio Meteorológico Nacional, es de $544,2 \mathrm{~mm}$. Esta información pluviométrica se vincula de manera estrecha con las determinaciones del contenido hídrico del suelo. Mientras para el día

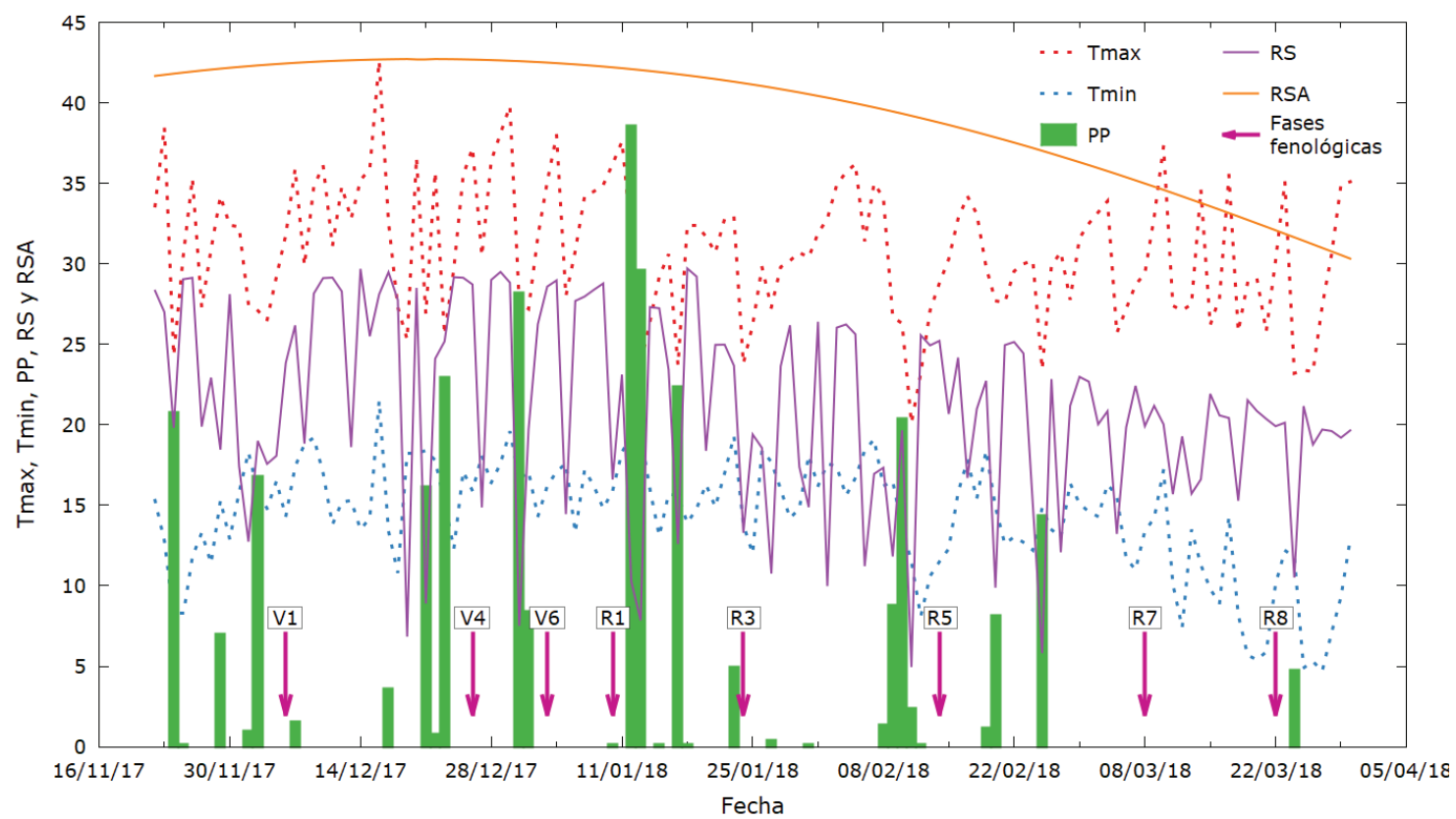

Figura 2. Condiciones meteorológicas durante las diferentes fases fenológicas del cultivo de soja en la campaña $2017-2018$ para el lote en estudio. (Temperatura máxima, Tmax, ${ }^{\circ} \mathrm{C}$; Temperatura mínima, Tmin, ${ }^{\circ} \mathrm{C}$; Precipitaciones, PP, mm; Radiación Solar Global, RS, $\mathrm{MJm}^{-2} \mathrm{~d}^{-1}$; Radiación Solar Astronómica, RSA, $\mathrm{MJm}^{-2} \mathrm{~d}^{-1}$ ) 
26/12/2017 el agua útil en el perfil arrojó un valor de $193 \pm 19,4 \mathrm{~mm}$, esto es un $81 \%$ del agua extraíble, el 6/02/2018 (fecha próxima a la adquisición de la imagen de Sentinel-2A), los valores de agua útil fueron de $18 \pm 10 \mathrm{~mm}$ lo que representó sólo el $8 \%$ del agua extraíble.

En la Figura 3 se presentan los valores absolutos del coeficiente de correlación lineal de Pearson ( $|r|)$ obtenidos para la relación entre el rendimiento del cultivo de soja y los diferentes índices de vegetación (IV) calculados de acuerdo a la diferencia (SD), el cociente (SR) y la diferencia normalizada (ND) de las distintas combinaciones de bandas de Sentinel-2A. De acuerdo a la Figura 3, se observa que los mayores valores de $|r|$ (próximos a 0,70) se obtuvieron cuando IV se calcula mediante la diferencia simple (SD), particularmente con las combinaciones de las bandas 2; 3; 4; 5; 11 ó 12 con las bandas 7; 8 u 8A. La mayor correlación con respecto al rendimiento de soja al final del ciclo corresponde a la diferencia entre las bandas 8 y $12(|r|=0,726)$. Los valores de $|r|$ más altos para los índices de vegetación derivados de diferencias simples consideran combinaciones de bandas correspondientes al espectro visible (2; 3 y 4), red edge (5) y SWIR $(11,12)$ con la última banda de red edge (7) y NIR (8 y 8 A).

Por su parte, los índices de vegetación derivados de cocientes (SR) y de diferencias normalizadas (ND) de combinaciones de bandas mostraron en cambio un desempeño menos adecuado cuando se los correlacionó con el rendimiento del cultivo de soja. El mayor valor de $|r|$ para los índices de vegetación de SR fue de 0,611 al combinar las bandas 8 y 11. Asimismo, con estas mismas bandas también se alcanzó el máximo valor absoluto del coeficiente de correlación $(0,605)$ cuando se utilizó la diferencia normalizada. Sin embargo, al emplear el NDVI el valor de $|r|$ fue de 0,436, sensiblemente menor que los montos anteriormente mencionados. Resultados similares fueron presentados por Tanaka et al. (2015), quienes encontraron los mayores valores de $\mathrm{R}^{2}$ al emplear las diferencias entre bandas de un espectroradiómetro para estimar el índice de área foliar en trigo de invierno, y valores de $\mathrm{R}^{2}$ más bajos para los cocientes y diferencias normalizadas de las bandas espectrales.

Los IV basados en la diferencia de bandas expresaron la respuesta del rendimiento tanto a indicadores basados en la absorción de la radiación debida a propiedades bioquímicas del canopeo (por ejemplo, clorofila), como a los cambios en la reflectancia debidas a estructura interna del dosel y la materia seca del cultivo, como así también a la absorción de la radiación por parte del agua presente en el canopeo. Por su parte, los IV de SR y ND se presentan más afectados en particular por la estructura, materia seca y contenido de agua del canopeo. La combinación de bandas NIR y SWIR, removería las variaciones producidas por la estructura interna y el contenido de materia seca de la hoja, siendo estos IV más sensibles al contenido hídrico del cultivo (Gu et al., 2007). De esa manera, las bandas relacionadas con el contenido de agua de la planta estarían indicando la influencia de diferentes niveles de estrés presentes en el lote sobre el rendimiento del cultivo de soja. Las propiedades de absorción del agua que determinan la reflectancia de la planta son el resultado de los procesos de vibración de los puentes $\mathrm{O}-\mathrm{H}$ de la molécula en las longitudes de onda próximas a los 975; 1175; 1450 y 1950 nm (Colombo et al., 2016).

A continuación, la Figura 4 presenta los mapas del lote en estudio elaborados a partir de los IV con mayores valores de $|r|$ para los cómputos mediante SD, SR y ND, como así también el mapa

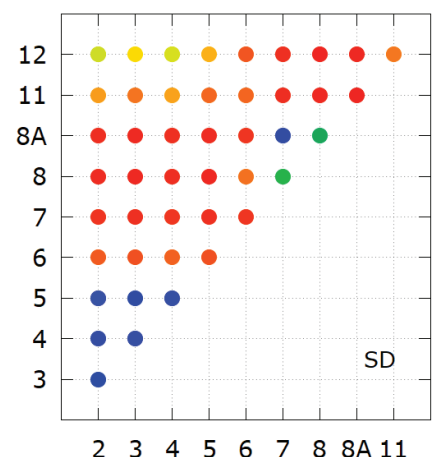

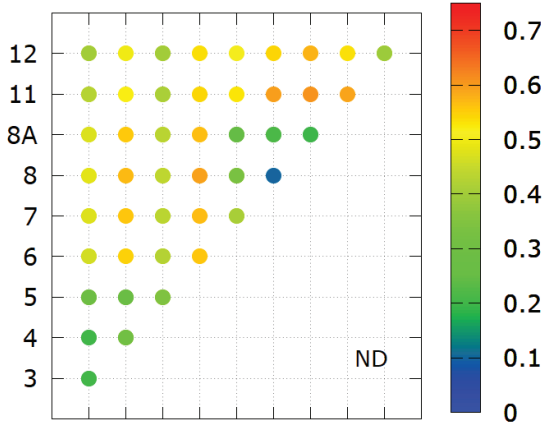

$\begin{array}{lllllllll}2 & 3 & 4 & 5 & 6 & 7 & 8 & 8 A & 11\end{array}$

Figura 3. Valores de absolutos del coeficiente de correlación lineal de Pearson entre el rendimiento del cultivo de soja y los diferentes índices de vegetación calculados a partir de las bandas de Sentinel 2A mediante diferencias (SD), cocientes (SR) y diferencias normalizadas (ND) (las especificaciones de cada banda se presentan en la Tabla 1) 


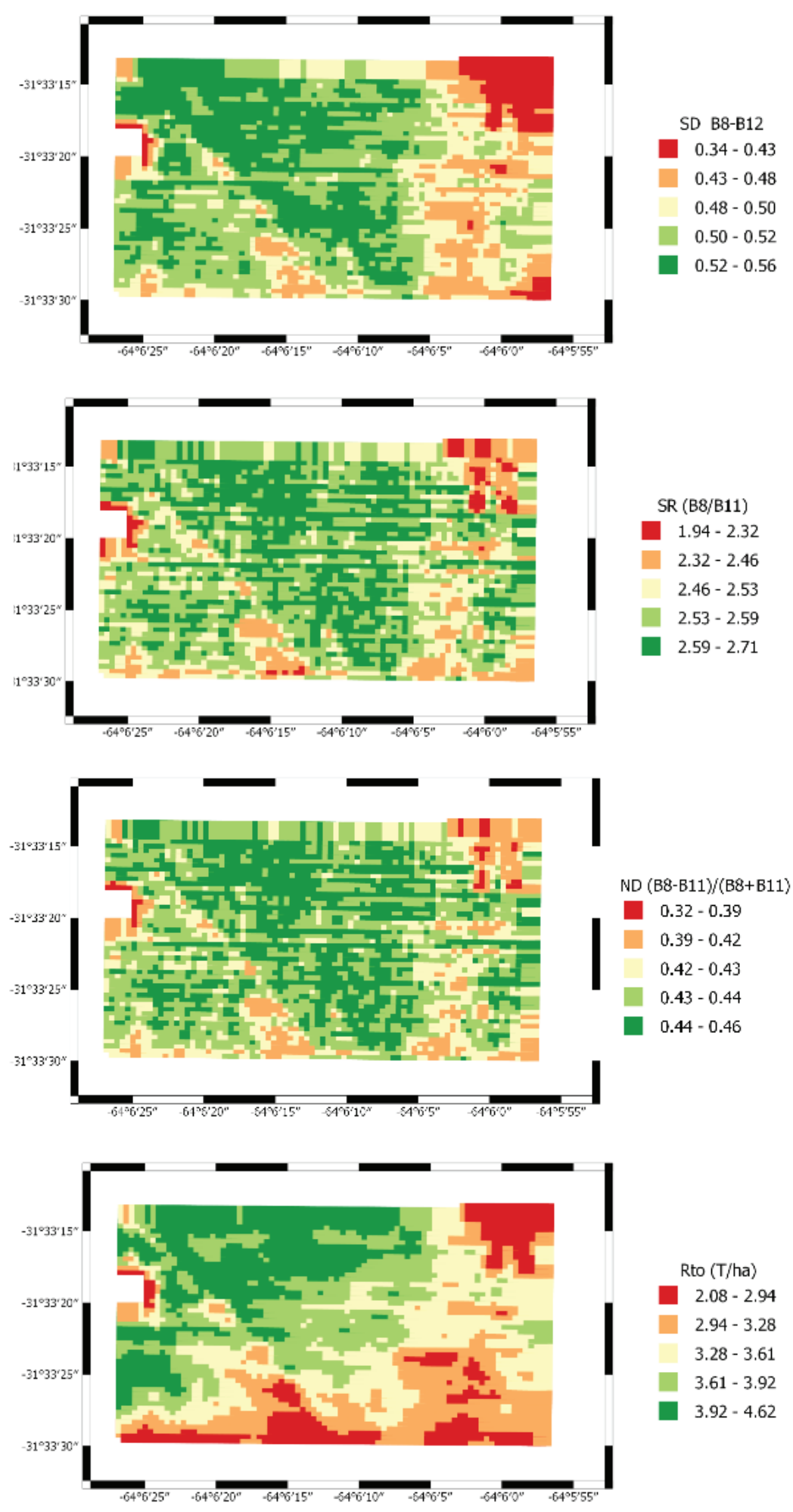

Figura 4. Mapas de los índices de vegetación de diferencia (SD), cociente (SR) y diferencias normalizadas (ND) con mayores valores absolutos del coeficiente de correlación lineal de Pearson y mapa de rendimiento (Rto) del cultivo de soja para el lote en estudio 
de rendimiento para facilitar la comparación visual de la información. En la Figura 4 puede observarse que el rendimiento de soja en el lote tuvo una fuerte dependencia espacial, probablemente como consecuencia de la intensa sequía registrada ese año. La productividad de la soja presentó un rango entre 2,08 y 4,62 t/ha, concentrándose los valores más elevados en el sector oeste y noroeste del lote, con excepción de la periferia de la casa, en tanto que los menores rendimientos se ubicaron en el sector sur $y$, en particular, en el sector noreste. La diferencia entre las bandas 8 y 12 es la que mejor copia la variabilidad espacial de los rendimientos alcanzados, siguiéndole el cociente y las diferencias normalizadas entre las bandas $8 \mathrm{y}$ 11 que no presentan marcadas diferencias entre sí.

En tal sentido, varios autores resaltaron la importancia de utilizar las bandas red edge y SWIR para mejorar la precisión de los procedimientos de clasificación e identificación de cultivos. Forkuor et al. (2017) señalaron la importancia de las bandas red edge en la clasificación de uso del suelo y tipos de cobertura en Burkina Faso empleando algoritmos de machine learning. Saito et al. (2019) destacaron la utilidad de las bandas SWIR de Sentinel-2 para discriminar arrozales de otros cultivos.

No obstante, Vuolo et al. (2018) señalan que, a los efectos de clasificar cultivos de diferente tipo en una región, un procedimiento de carácter multitemporal mitiga de manera eficiente los efectos negativos observados cuando se utiliza la adquisición de una sola fecha que incorpora ventanas temporales subóptimas. Schultz et al. (2015) clasificaron diferentes cultivos, entre ellos soja, en el sureste de Brasil, considerando solamente las bandas roja, NIR y SWIR de dos imágenes Landsat, selección que justificaron por la baja correlación inter-banda. Immitzer et al. (2016) confirmaron en su estudio la importancia de utilizar bandas de red edge y SWIR en la clasificación de distintos tipos de bosques y campos agrícolas a partir de imágenes Sentinel-2.

Ramoelo et al. (2015), por su parte, empleando un algoritmo de Random Forest, determinaron que las bandas de Sentinel-2 más importantes para predecir nitrógeno foliar en la sabana africana fueron la red edge $(705 \mathrm{~nm})$ y las dos SWIR (1610 y $2190 \mathrm{~nm})$. De esta forma, con los datos de Sentinel-2 se abre una gran oportunidad para efectuar el monitoreo más detallado de cultivos por teledetección, haciendo el seguimiento en particular de estreses de distinta naturaleza, como así también para aprovechar las ventajas del manejo sitio-específico.
A partir de modelos lineales simples se estimó el rendimiento del cultivo de soja empleando Ios IV que obtuvieron mejores valores absolutos del coeficiente de regresión. Los valores de pendiente y ordenada al origen para todos ellos son estadísticamente significativos. También se obtuvieron los valores de RMSE y \%RMSE, que presentan un rango entre $0,291-0,334$ t/ha y 8,31 - 9,53\%, respectivamente (Tabla 2). En la Figura 5 se muestra el scatterplot del índice estimado a partir de las diferencias entre las bandas 8 y 12, que presentó mejor desempeño al estimar el rendimiento del cultivo de soja. Resultados similares fueron señalados por Bolton y Friedl (2013) quienes encontraron que los índices NDVI y EVI2 a los 80 días del establecimiento del cultivo, presentaron un desempeño similar en predecir el rendimiento de soja a nivel de condados en USA, con valores de $r$ de 0,83 y 0,84 , respectivamente y valores de RMSE entre 0,254 y 0,456 t/ha.

Tabla 2. Coeficientes de pendiente (a) y ordenada al origen (b) de los modelos lineales para estimar el rendimiento de soja a partir de los índices de vegetación de diferencia (SD), cociente (SR) y diferencias normalizadas (ND), y los estadísticos raíz cuadrada del error cuadrático medio (RMSE) y su valor porcentual respecto del valor medio observado (\%RMSE)

\begin{tabular}{ccccc}
\hline Índice de vegetación & a & $b$ & $\begin{array}{c}\text { RMSE } \\
(\mathrm{t} / \mathrm{ha})\end{array}$ & $\begin{array}{c}\% \mathrm{RMSE} \\
(\%)\end{array}$ \\
\hline $\begin{array}{c}\text { SD } \\
(\mathrm{B} 8-\mathrm{B} 12) \\
\text { SR } \\
(\mathrm{B} 8 / \mathrm{B} 11)\end{array}$ & $9,962^{*}$ & $-1,518^{*}$ & 0,291 & 8,31 \\
$\mathrm{ND}$ & $3,522^{*}$ & $-5,471^{*}$ & 0,332 & 9,47 \\
$\begin{array}{c}\text { (B8-B11)/(B8+B11) } \\
\text { (B8 }\end{array}$ & $21,049^{*}$ & $-5,675^{*}$ & 0,334 & 9,53 \\
\hline
\end{tabular}

* Significativos al $5 \%$ de probabilidad.

Frecuencia

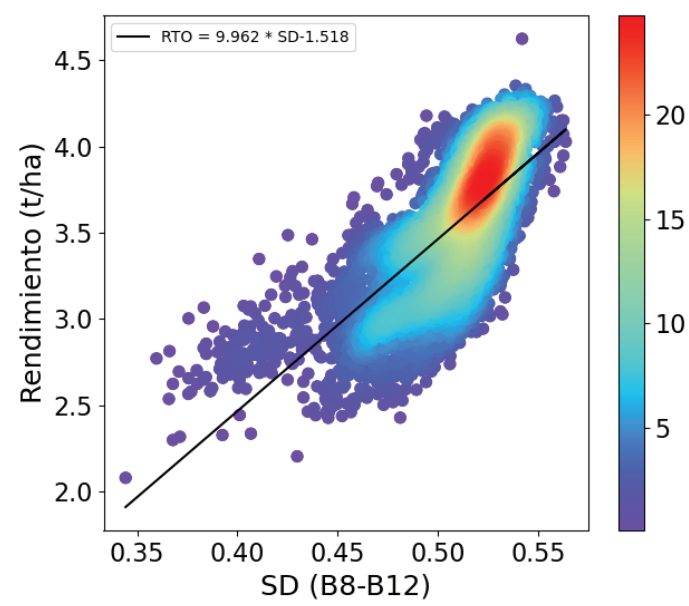

Figura 5. Scatterplot del índice de vegetación de la diferencias entre las bandas B8 y B12 vs. el rendimiento del cultivo de soja, con código de colores de acuerdo a la frecuencia 
La Figura 6 presenta los mapas de rendimientos estimados con un modelo lineal a partir de los índices de vegetación de diferencia (SD), cociente (SR) y diferencias normalizadas (ND) de las bandas que presentaron los mayores valores de $r$ y sus respectivos errores de estimación. En general los tres índices presentan una distribución similar de los rendimientos estimados, con los menores valores concentrados en la porción este del lote, particularmente en la noreste, en tanto que los valores más altos se encuentran en la parte central y noroeste del lote. El índice de la diferencia de bandas predice valores mucho más elevados (entre 3,48 y 4,10 t/ha) concentrados en la porción noreste del lote, a diferencia de los índices de cociente y de la diferencia normalizada que estiman pocos píxeles con este rango de rendimientos.

Con respecto a los errores que producen los modelos, los tres índices considerados presentan subestimaciones en la porción noroeste de la parcela, en tanto que los menores errores se
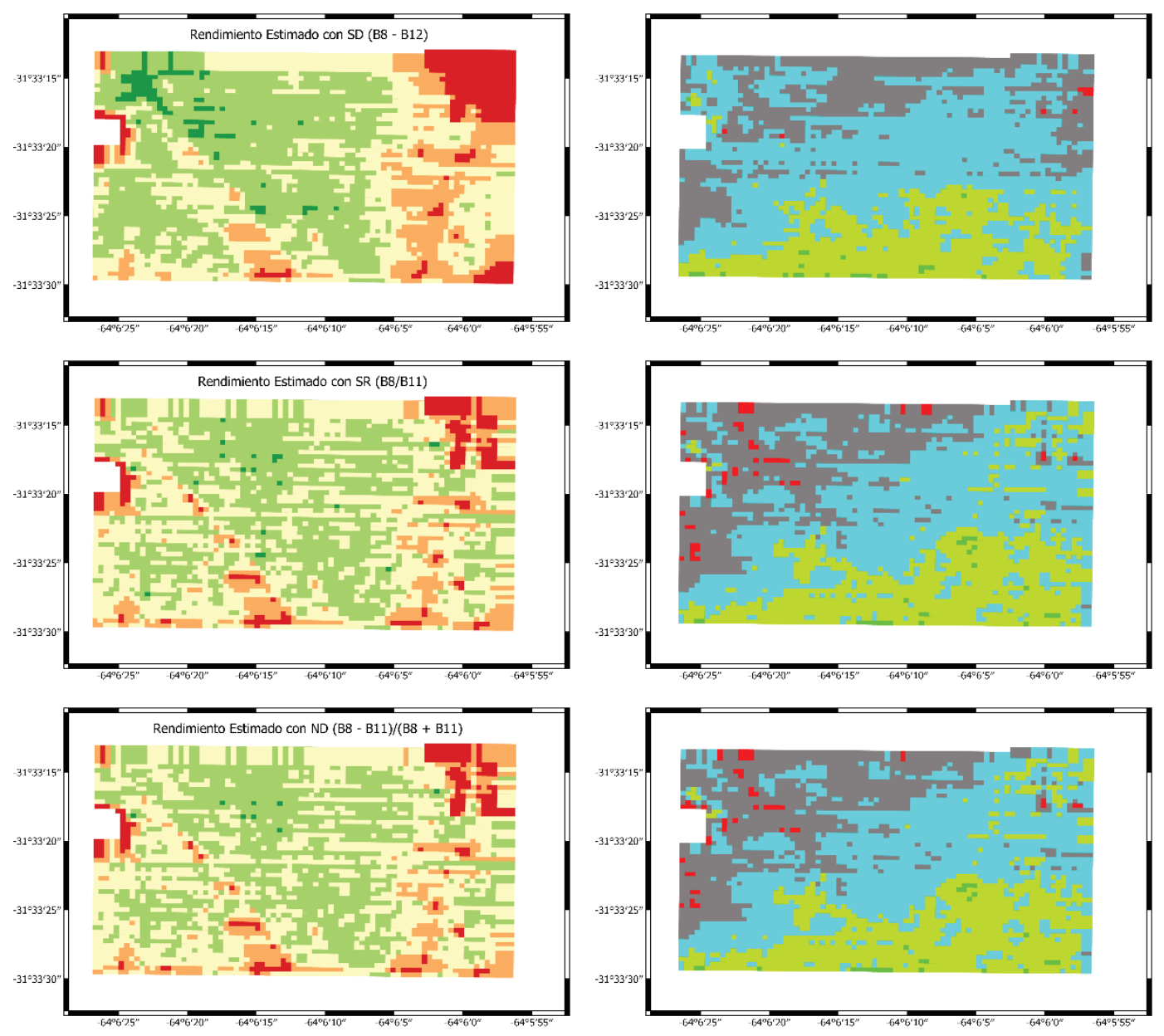

Referencias:

Rendimientos (t/ha)

$$
\begin{array}{r}
\square 1,00 \text { a } 1,62 \\
1,62 \text { a } 2,24 \\
2,24 \text { a } 2,86 \\
2,86 \text { a } 3,48 \\
-3,48 \text { a } 4,10
\end{array}
$$

Errores (t/ha)
$\square-1,15$ a $-0,69$
$\square-0,69$ a $-0,23$
$-0,23$ a 0,23
0,23 a 0,69
0,69 a 1,15

Figura 6. Mapas de rendimientos del cultivo de soja estimados a partir de modelos lineales considerando los índices de vegetación de diferencia (SD), cociente (SR) y diferencia normalizada (ND) (izquierda) con sus respectivos errores de estimación (derecha) 
encuentran en una franja que corre desde el suroeste al noreste del lote, quedando la porción sureste con sobreestimaciones del rendimiento. En particular, las estimaciones producidas a partir de la diferencia de bandas presentan menor cantidad de errores extremos entre 1,15 y 0,69 t/ha o entre $-1,15$ y $-0,69 \mathrm{t} / \mathrm{ha}$.

\section{CONCLUSIONES}

Diferentes combinaciones de las bandas NIR y SWIR de Sentinel-2A se mostraron estrechamente vinculadas con el rendimiento de soja, pudiendo realizar una estimación relativamente precisa de la productividad del cultivo al comienzo de las etapas reproductivas, con un buen anticipo respecto a la fecha de cosecha. El índice que se obtuvo a partir de la diferencia entre las bandas 8 y 12 de Sentinel$2 \mathrm{~A}$ es el que mostró el mejor desempeño predictivo de la productividad final, alcanzando valores elevados de correlación, y permitiendo reproducir con suficiente precisión la variabilidad espacial de los rendimientos en el lote. De esta forma, la información que se genera a partir de los datos de Sentinel-2A presenta una gran potencialidad a los efectos de implementar un manejo sitioespecífico, tanto para clasificar áreas homogéneas en un lote, como a fin de reconocer diferencias de productividad entre distintos sectores.

\section{AGRADECIMIENTOS}

Este trabajo forma parte del proyecto PIP 33620180100318CB "Determinación de la productividad potencial y la brecha de rendimientos de cultivos en la región central de Córdoba orientada al manejo sitio-específico a partir de datos radiométricos y de termometría infrarroja" financiado por la Secretaría de Ciencia y Técnica (SECYT) de la Universidad Nacional de Córdoba.

\section{BIBLIOGRAFÍA}

Allen, R. G., Pereira, L. S., Raes, D. y Smith, M. (Eds.) (1998). Crop evapotranspiration: Guide-lines for computing crop water requirements - FAO Irrigation and Drainage Paper 56. Organización de las Naciones Unidas para la Alimentación y la Agricultura (FAO).

Anselin, L., Syabri, I. y Kho Y. (2006). GeoDa: An Introduction to Spatial Data Analysis. Geographical Analysis, 38, 5-22. https://doi.org/10.1111/j.00167363.2005.00671.x

Battude, M., Al Bitar, A., Morin, D., Cros, J., Huc, M., Sicre,
C. M., Le Dantec, V. y Demarez, V. (2016). Estimating maize biomass and yield over large areas using high spatial and temporal resolution Sentinel-2 like remote sensing data. Remote Sensing of Environment, 184, 668-681. https://doi.org/10.1016/j.rse.2016.07.030

Bolton, D. K. y Friedl, M. A. (2013). Forecasting crop yield using remotely sensed vegetation indices and crop phenology metrics. Agricultural and Forest Meteorology, 173, 74-84. https://doi.org/10.1016/j. agrformet.2013.01.007

Bottega, E. L., de Queiroz, D. M., de Assis de Carvalho Pinto, F., Valente, D. S. M. y de Souza, C. M. A. (2017). Precision agriculture applied to soybean: Part IIISpatial and temporal variability of yield. Australian Journal of Crop Science, 11(7), 799-805. https://doi. org/10.21475/ajcs.17.11.07.pne383

Clevers, J. G. y Gitelson, A. A. (2013). Remote estimation of crop and grass chlorophyll and nitrogen content using red-edge bands on Sentinel-2 and-3. International Journal of Applied Earth Observation and Geoinformation, 23, 344-351. https://doi.org/10.1016/j. jag.2012.10.008

Colombo, R., Busetto, L., Meroni, M., Rossini, M. y Panigada, C (2016). Optical remote sensing of vegetation water content. En: P. S. Thenkabail, J. G. Lyon y A. Huete (Eds.), Hyperspectral Remote Sensing of Vegetation (pp. 227-244). CRC Press.

Crusiol, L. G. T., Carvalho, J. D. F. C., Sibaldelli, R. N. R., Neiverth, W., do Rio, A., Ferreira, L. C., Procópio, S., Mertz-Henning, L. M., Nepomuceno, A. L., Neumaier, N. y Farias, J. R. B. (2017). NDVI variation according to the time of measurement, sampling size, positioning of sensor and water regime in different soybean cultivars. Precision agriculture, 18(4), 470-490. https://doi. org/10.1007/s11119-016-9465-6

Dardanelli, J. L., Bachmeier, O. A., Sereno, R. y Gil, R. (1997). Rooting depth and soil water extraction patterns of different crops in a silty loam Haplustoll. Field Crops Research, 54, 29-38. https://doi.org/10.1016/S03784290(97)00017-8

Fehr, W. R. y Caviness, C. E. (1977). Stages of soybean development. (Informe Especial n $n^{\circ} 80$ de Agriculture and Home Economics Experiment Station). lowa State University.

Forkuor, G., Dimobe, K., Serme, I. y Tondoh, J. E. (2017). Landsat-8 vs. Sentinel-2: examining the added value of sentinel-2's red-edge bands to land-use and landcover mapping in Burkina Faso. GIScience \& remote sensing, 55(3), 331-354. https://doi.org/10.1080/1548 1603.2017.1370169

Frampton, W. J., Dash, J., Watmough, G. y Milton, E. J. (2013). Evaluating the capabilities of Sentinel-2 for quantitative estimation of biophysical variables in vegetation. ISPRS journal of photogrammetry and 
remote sensing, 82, 83-92. https://doi.org/10.1016/j isprsjprs.2013.04.007

Gu, Y., Brown, J. F., Verdin, J. P. y Wardlow, B. (2007). A five year analysis of MODIS NDVI and NDWI for grassland drought assessment over the central Great Plains of the United States. Geophysical Research Letters, 34 (6). https://doi.org/10.1029/2006GL029127

Haboudane, D., Miller, J. R., Pattey, E., Zarco-Tejada, P. J. y Strachan, I. B. (2004). Hyperspectral vegetation indices and novel algorithms for predicting green LAI of crop canopies: Modeling and validation in the context of precision agriculture. Remote sensing of environment, 90(3), 337-352. https://doi:10.1016/j. rse.2003.12.013

Immitzer, M., Vuolo, F. y Atzberger, C. (2016). First experience with Sentinel-2 data for crop and tree species classifications in central Europe. Remote Sensing, 8(3), 166. https://doi:10.3390/rs8030166

Khanal, S., Fulton, J. y Shearer, S. (2017). An overview of current and potential applications of thermal remote sensing in precision agriculture. Computers and Electronics in Agriculture, 139, 22-32. https://doi. org/10.1016/j.compag.2017.05.001

Kovar, M., Brestic, M., Sytar, O., Barek, V., Hauptvogel, P. y Zivcak, M. (2019). Evaluation of Hyperspectral Reflectance Parameters to Assess the Leaf Water Content in Soybean. Water, 11(3), 443. https://doi. org/10.3390/w11030443

Li, M., Chu, R., Yu, Q., Islam, A., Chou, S. y Shen, S. (2018). Evaluating Structural, Chlorophyll-Based and Photochemical Indices to Detect Summer Maize Responses to Continuous Water Stress. Water, 10(4), 500. https://doi.org/10.3390/w10040500

Louis, J., Debaecker, V., Pflug, B., Main-Knorn, M., Bieniarz, J., Mueller-Wilm, U., Cadau, E. y Gascon, F. (Mayo, 2016). Sentinel-2 SEN2COR: L2A processor for users. En Proceedings of the Living Planet Symposium (pp. 9-13). Prague, Czech Republic. European Space Agency.

Lu, S., Lu, X., Zhao, W., Liu, Y., Wang, Z. y Omasa, K. (2015). Comparing vegetation indices for remote chlorophyll measurement of white poplar and Chinese elm leaves with different adaxial and abaxial surfaces. Journal of experimental botany, 66(18), 5625-5637. https://doi.org/10.1093/jxb/erv270

Mladenova, I. E., Bolten, J. D., Crow, W. T., Anderson, M. C., Hain, C. R., Johnson, D. M. y Mueller, R. (2017). Intercomparison of soil moisture, evaporative stress, and vegetation indices for estimating corn and soybean yields over the US. IEEE Journal of Selected Topics in Applied Earth Observations and Remote Sensing, 10(4), 1328-1343. https://doi.org/10.1109/ JSTARS.2016.2639338

Nigam, R., Vyas, S. S., Bhattacharya, B. K., Oza, M. P. y
Manjunath, K. R. (2017). Retrieval of regional LAI over agricultural land from an Indian geostationary satellite and its application for crop yield estimation. Journal of Spatial Science, 62, 103-125. https://doi.org/10.1080/ 14498596.2016 .1220872

Peng, Y., Nguy-Robertson, A., Arkebauer, T. y Gitelson, A. (2017). Assessment of canopy chlorophyll content retrieval in maize and soybean: implications of hysteresis on the development of generic algorithms. Remote Sensing, 9(3), 226. https://doi.org/10.3390/ rs9030226

Petersen, L. (2018). Real-Time Prediction of Crop Yields from MODIS Relative Vegetation Health: A ContinentWide Analysis of Africa. Remote Sensing, 10(11), 1726. https://doi.org/10.3390/rs10111726

Ramoelo, A., Cho, M., Mathieu, R. y Skidmore, A. K. (2015). Potential of Sentinel-2 spectral configuration to assess rangeland quality. Journal of applied remote sensing, 9, 094096. https://doi:10.1117/1.JRS.9.094096

Rollán, A. D. C. y Bachmeier, O. A. (2014). Compactación y retención hídrica en Haplustoles de la provincia de Córdoba (Argentina) bajo siembra directa. Agriscientia, 31, 1-10. https://doi.org/10.31047/1668.298x.v31. $\mathrm{n} 1.9835$

Rouse, J. W., Haas, R. H., Schell, J. A. y Deering, D. W. (1973). Monitoring vegetation systems in the Great Plains with ERTS. En Proceedings of the Third Earth Resources Technology Satellite Symposium (pp. 309317). Washington, DC. NASA.

Saito, G., Uto, K., Yonezawa, C., Sasaki, Y., Yoshino, K., Oda, K., Sato, J., Oyoshi, K. y Mizukami, Y. (Mayo 2019). Determination of planting crops using satellite data at Shonai plain in Japan. En IOP Conference Series: Earth and Environmental Science (Vol. 284, 012009). IOP Publishing.

Schultz, B., Immitzer, M., Formaggio, A., Sanches, I., Luiz, A. y Atzberger, C. (2015). Self-guided segmentation and classification of multi-temporal Landsat 8 images for crop type mapping in southeastern Brazil. Remote Sensing, 7(11), 14482-14508. https://doi.org/10.3390/ rs71114482

Tanaka, S., Kawamura, K., Maki, M., Muramoto, Y., Yoshida, K. y Akiyama, T. (2015). Spectral index for quantifying leaf area index of winter wheat by field hyperspectral measurements: a case study in Gifu prefecture, central Japan. Remote Sensing, 7(5), 5329-5346. https://doi.org/10.3390/rs70505329

Viña, A., Gitelson, A. A., Rundquist, D. C., Keydan, G., Leavitt, B. y Schepers, J. (2004). Monitoring maize (Zea mays L.) phenology with remote sensing. Agronomy Journal, 96(4), 1139-1147. https://doi. org/10.2134/agronj2004.1139

Viña, A. y Gitelson, A. A. (2005). New developments in the remote estimation of the fraction of absorbed 
photosynthetically active radiation in crops. Geophysical Research Letters, 32(17). https://doi. org/10.1029/2005GL023647

Vuolo, F., Neuwirth, M., Immitzer, M., Atzberger, C. y Ng, W. (2018). How much does multi-temporal Sentinel-2 data improve crop type classification? International Journal of Applied Earth Observation and Geoinformation, 72, 122-130. https://doi.org/10.1016/j.jag.2018.06.007

Xie, Q., Dash, J., Huete, A., Jiang, A., Yin, G., Ding, Y., Peng, D., Halla, C. C., Brown, L., Shi, Y., Ye, H., Dong, Y. y Huang W. (2019). Retrieval of crop biophysical parameters from Sentinel-2 remote sensing imagery. International Journal of Applied Earth Observation and
Geoinformation, 80, 187-195. https://doi.org/10.1016/j. jag.2019.04.019

Xu, C., Qu, J. J., Hao, X., Cosh, M. H., Zhu, Z. y Gutenberg, L. (2020). Monitoring crop water content for corn and soybean fields through data fusion of MODIS and Landsat measurements in lowa. Agricultural Water Management, 227, 105844. https://doi.org/10.1016/j. agwat.2019.105844

Xue, J. y Su, B. (2017). Significant remote sensing vegetation indices: a review of developments and applications. Journal of Sensors, 1353691. https://doi. org/10.1155/2017/1353691 